\title{
CONDITIONAL INTEGRAL TRANSFORMS AND CONVOLUTIONS OF BOUNDED FUNCTIONS ON AN ANALOGUE OF WIENER SPACE
}

\author{
DONG Hyun Cho*
}

\begin{abstract}
Let $C[0, t]$ denote the function space of all real-valued continuous paths on $[0, t]$. Define $X_{n}: C[0, t] \rightarrow \mathbb{R}^{n+1}$ and $X_{n+1}$ : $C[0, t] \rightarrow \mathbb{R}^{n+2}$ by $X_{n}(x)=\left(x\left(t_{0}\right), x\left(t_{1}\right), \cdots, x\left(t_{n}\right)\right)$ and $X_{n+1}(x)=$ $\left(x\left(t_{0}\right), x\left(t_{1}\right), \cdots, x\left(t_{n}\right), x\left(t_{n+1}\right)\right)$, where $0=t_{0}<t_{1}<\cdots<t_{n}<$ $t_{n+1}=t$. In the present paper, using simple formulas for the conditional expectations with the conditioning functions $X_{n}$ and $X_{n+1}$, we evaluate the $L_{p}(1 \leq p \leq \infty)$-analytic conditional FourierFeynman transforms and the conditional convolution products of the functions which have the form

$$
\int_{L_{2}[0, t]} \exp \{i(v, x)\} d \sigma(v) \int_{\mathbb{R}^{r}} \exp \left\{i \sum_{j=1}^{r} z_{j}\left(v_{j}, x\right)\right\} d \rho\left(z_{1}, \cdots, z_{r}\right)
$$

for $x \in C[0, t]$, where $\left\{v_{1}, \cdots, v_{r}\right\}$ is an orthonormal subset of $L_{2}[0, t]$ and $\sigma$ and $\rho$ are the complex Borel measures of bounded variations on $L_{2}[0, t]$ and $\mathbb{R}^{r}$, respectively. We then investigate the inverse transforms of the function with their relationships and finally prove that the analytic conditional Fourier-Feynman transforms of the conditional convolution products for the functions, can be expressed in terms of the products of the conditional FourierFeynman transforms of each function.
\end{abstract}

Received January 12, 2013; Accepted April 04, 2013.

2010 Mathematics Subject Classification: Primary 28C20; Secondary 44A35, 60G15, 60H05.

Key words and phrases: analogue of Wiener measure, analytic conditional Feynman integral, analytic conditional Fourier-Feynman transform, analytic conditional Wiener integral, conditional convolution product.

This research was supported by Basic Science Research Program through the National Research Foundation of Korea(NRF) funded by the Ministry of Education, Science and Technology(2012-0002477). 


\section{Introduction and preliminaries}

Let $C_{0}[0, t]$ denote the Wiener space, that is, the space of real-valued continuous functions $x$ on the closed interval $[0, t]$ with $x(0)=0$. Chang and Skoug ([3]) introduced the concepts of conditional Fourier-Feynman transform and conditional convolution product on the Wiener space $C_{0}[0, t]$. In that paper, they examined the effects that drift has on the conditional Fourier-Feynman transform, the conditional convolution product, and various relationships that occur between them. Further works were studied by the author and his coauthors $([2,9])$. In fact, they $([2])$ introduced the $L_{1}$-analytic conditional Fourier-Feynman transform and the conditional convolution product over Wiener paths in abstract Wiener space and then, established their relationships between them of certain cylinder type functions. The author $([9])$ extended the relationships between the conditional convolution product and the $L_{p}(1 \leq p \leq 2)$-analytic conditional Fourier-Feynman transform of the same kind of cylinder functions. Moreover, on $C[0, t]$, the space of the real-valued continuous paths on $[0, t]$, Kim ([12]) extended the relationships between the conditional convolution product and the $L_{p}(1 \leq p \leq \infty)$-analytic conditional Fourier-Feynman transform of the functions in a Banach algebra which corresponds to the CameronStorvick's Banach algebra $\mathcal{S}([1])$. The author $([4,5,6])$ established several relationships between the $L_{p}(1 \leq p \leq \infty)$-analytic conditional Fourier-Feynman transforms and the conditional convolution products of the cylinder functions on $C[0, t]$. In particular, he $([4,5])$ derived an evaluation formula for the $L_{p}$-analytic conditional Fourier-Feynman transforms and the conditional convolution products of the same cylinder functions with the conditioning functions $X_{n}: C[0, t] \rightarrow \mathbb{R}^{n+1}$ and $X_{n+1}: C[0, t] \rightarrow \mathbb{R}^{n+2}$ given by $X_{n}(x)=\left(x\left(t_{0}\right), x\left(t_{1}\right), \cdots, x\left(t_{n}\right)\right)$ and $X_{n+1}(x)=\left(x\left(t_{0}\right), x\left(t_{1}\right), \cdots, x\left(t_{n}\right), x\left(t_{n+1}\right)\right)$, where $0=t_{0}<t_{1}<\cdots<$ $t_{n}<t_{n+1}=t$ is a partition of $[0, t]$, and then, derived their relationships. Note that $X_{n}$ is independent of the present time $t$ while $X_{n+1}$ is wholly dependent on the present time. In this paper, we further develop the relationships in $([4,5,12])$ on the more generalized space $\left(C[0, t], w_{\varphi}\right)$, an analogue of the Wiener space associated with the probability measure $\varphi$ on the Borel class $\mathcal{B}(\mathbb{R})$ of $\mathbb{R}([11,13,14])$. For the conditioning functions $X_{n}$ and $X_{n+1}$, we proceed to study the relationships between the conditional convolution products and the analytic conditional FourierFeynman transforms of bounded functions defined on $C[0, t]$. In fact, using simple formulas for the conditional $w_{\varphi}$-integrals given $X_{n}$ and $X_{n+1}$, 
we evaluate the $L_{p}(1 \leq p \leq \infty)$-analytic conditional Fourier-Feynman transforms and the conditional convolution products for the functions of the form

$$
\int_{L_{2}[0, t]} \exp \{i(v, x)\} d \sigma(v) \int_{\mathbb{R}^{r}} \exp \left\{i \sum_{j=1}^{r} z_{j}\left(v_{j}, x\right)\right\} d \rho\left(z_{1}, \cdots, z_{r}\right)
$$

for $w_{\varphi}$-a.e. $x \in C[0, t]$, where $\left\{v_{1}, \cdots, v_{r}\right\}$ is an orthonormal set in $L_{2}[0, t], \sigma$ is a complex Borel measure of bounded variation on $L_{2}[0, t]$ and $\rho$ is a bounded complex Borel measure on $\mathbb{R}^{r}$. We then investigate various relationships between the conditional Fourier-Feynman transforms and the conditional convolution products of the functions given by (1.1). Finally, we show that the $L_{p}$-analytic conditional FourierFeynman transform of the conditional convolution product for the functions $\Psi_{1}$ and $\Psi_{2}$ given by (1.1), can be expressed by the formula

$$
\begin{aligned}
& T_{q}^{(p)}\left[\left[\left(\Psi_{1} * \Psi_{2}\right)_{q} \mid X_{n}\right]\left(\cdot, \vec{\xi}_{n}\right) \mid X_{n}\right]\left(y, \vec{\zeta}_{n}\right) \\
& =\left[T_{q}^{(p)}\left[\Psi_{1} \mid X_{n}\right]\left(\frac{1}{\sqrt{2}} y, \frac{1}{\sqrt{2}}\left(\vec{\zeta}_{n}+\vec{\xi}_{n}\right)\right)\right] \\
& \quad \times\left[T_{q}^{(p)}\left[\Psi_{2} \mid X_{n}\right]\left(\frac{1}{\sqrt{2}} y, \frac{1}{\sqrt{2}}\left(\vec{\zeta}_{n}-\vec{\xi}_{n}\right)\right)\right]
\end{aligned}
$$

for a nonzero real $q, w_{\varphi}$-a.e. $y \in C[0, t]$ and $P_{X_{n}}$-a.e. $\vec{\xi}_{n}, \vec{\zeta}_{n} \in \mathbb{R}^{n+1}$. Thus the analytic conditional Fourier-Feynman transform of the conditional convolution product for the functions, can be interpreted as the product of the analytic conditional Fourier-Feynman transform of each function.

Let $\mathbb{C}, \mathbb{C}_{+}$and $\mathbb{C} \sim$ denote the set of complex numbers, the set of complex numbers with positive real parts and the set of nonzero complex numbers with nonnegative real parts, respectively.

Let $C=C[0, t]$ be the space of all real-valued continuous functions on the closed interval $[0, t]$ with its Borel class $\mathcal{B}(C[0, t])$. For a probability measure $\varphi$ on $(\mathbb{R}, \mathcal{B}(\mathbb{R}))$, let $w_{\varphi}$ be an analogue of the Wiener measure on $\mathcal{B}(C[0, t])$ associated with $\varphi([11,13,14])$. Let $F: C[0, t] \rightarrow \mathbb{C}$ be integrable and $X$ be a random vector on $C[0, t]$ assuming that the value space of $X$ is a normed space equipped with the Borel $\sigma$-algebra. Then, we have the conditional expectation $E[F \mid X]$ of $F$ given $X$ from a well known probability theory. Furthermore, there exists a $P_{X}$-integrable $\mathbb{C}$ valued function $\psi$ on the value space of $X$ such that $E[F \mid X](x)=(\psi \circ$ $X)(x)$ for $w_{\varphi}$-a.e. $x \in C[0, t]$, where $P_{X}$ is the probability distribution 
of $X$. The function $\psi$ is called the conditional $w_{\varphi}$-integral of $F$ given $X$ and it is also denoted by $E[F \mid X]$.

Throughout this paper, let $0=t_{0}<t_{1}<\cdots<t_{n}<t_{n+1}=t$ be a partition of $[0, t]$, where $n$ is a positive integer. For any $x$ in $C[0, t]$, define the polygonal function $[x]$ of $x$ by

$$
\begin{aligned}
{[x](s)=} & \sum_{j=1}^{n+1} \chi_{\left(t_{j-1}, t_{j}\right]}(s)\left(\frac{t_{j}-s}{t_{j}-t_{j-1}} x\left(t_{j-1}\right)+\frac{s-t_{j-1}}{t_{j}-t_{j-1}} x\left(t_{j}\right)\right) \\
& +\chi_{\left\{t_{0}\right\}}(s) x\left(t_{0}\right)
\end{aligned}
$$

for $s \in[0, t]$, where $\chi_{\left(t_{j-1}, t_{j}\right]}$ and $\chi_{\left\{t_{0}\right\}}$ denote the indicator functions. Similarly, for $\vec{\xi}_{n+1}=\left(\xi_{0}, \xi_{1}, \cdots, \xi_{n+1}\right) \in \mathbb{R}^{n+2}$, define the polygonal function $\left[\vec{\xi}_{n+1}\right]$ of $\vec{\xi}_{n+1}$ by $(1.2)$, where $x\left(t_{j}\right)$ is replaced by $\xi_{j}$ for $j=$ $0,1, \cdots, n+1$. Let $X_{n+1}: C[0, t] \rightarrow \mathbb{R}^{n+2}$ and $X_{n}: C[0, t] \rightarrow \mathbb{R}^{n+1}$ be given by

$$
X_{n+1}(x)=\left(x\left(t_{0}\right), x\left(t_{1}\right), \cdots, x\left(t_{n}\right), x\left(t_{n+1}\right)\right)
$$

and

$$
X_{n}(x)=\left(x\left(t_{0}\right), x\left(t_{1}\right), \cdots, x\left(t_{n}\right)\right),
$$

respectively. For a function $F: C[0, t] \rightarrow \mathbb{C}$ and $\lambda>0$, let $F^{\lambda}(x)=$ $F\left(\lambda^{-\frac{1}{2}} x\right), X_{n+1}^{\lambda}(x)=X_{n+1}\left(\lambda^{-\frac{1}{2}} x\right)$ and $X_{n}^{\lambda}(x)=X_{n}\left(\lambda^{-\frac{1}{2}} x\right)$, where $X_{n+1}$ and $X_{n}$ are given by (1.3) and (1.4), respectively. Suppose that $E\left[F^{\lambda}\right]$ exists for each $\lambda>0$. By the definition of the conditional $w_{\varphi^{-}}$ integral and the equation (6) of Theorem 2.9 in [8],

$$
E\left[F^{\lambda} \mid X_{n+1}^{\lambda}\right]\left(\vec{\xi}_{n+1}\right)=E\left[F\left(\lambda^{-\frac{1}{2}}(x-[x])+\left[\vec{\xi}_{n+1}\right]\right)\right]
$$

for $P_{X_{n+1}^{\lambda}}$-a.e. $\vec{\xi}_{n+1} \in \mathbb{R}^{n+2}$, where $P_{X_{n+1}^{\lambda}}$ is the probability distribution of $X_{n+1}^{\lambda}$ on $\left(\mathbb{R}^{n+2}, \mathcal{B}\left(\mathbb{R}^{n+2}\right)\right)$. Throughout this paper, for $y \in C[0, t]$ let

$$
I_{F}^{\lambda}\left(y, \vec{\xi}_{n+1}\right)=E\left[F\left(y+\lambda^{-\frac{1}{2}}(x-[x])+\left[\vec{\xi}_{n+1}\right]\right)\right]
$$

unless otherwise specified, where the expectation is taken over the variable $x$. Moreover, we can obtain from (2.6) of Theorem 2.5 in [7]

$$
\begin{aligned}
& E\left[F^{\lambda} \mid X_{n}^{\lambda}\right]\left(\vec{\xi}_{n}\right) \\
& =\left[\frac{\lambda}{2 \pi\left(t-t_{n}\right)}\right]^{\frac{1}{2}} \int_{\mathbb{R}} I_{F}^{\lambda}\left(0, \vec{\xi}_{n+1}\right) \exp \left\{-\frac{\lambda}{2} \frac{\left(\xi_{n+1}-\xi_{n}\right)^{2}}{t-t_{n}}\right\} d \xi_{n+1}
\end{aligned}
$$

for $P_{X_{n}^{\lambda}}$-a.e. $\vec{\xi}_{n}=\left(\xi_{0}, \xi_{1}, \cdots, \xi_{n}\right) \in \mathbb{R}^{n+1}$, where $\vec{\xi}_{n+1}=\left(\xi_{0}, \xi_{1}, \cdots, \xi_{n}\right.$, $\left.\xi_{n+1}\right)$ for $\xi_{n+1} \in \mathbb{R}$ and $P_{X_{n}^{\lambda}}$ is the probability distribution of $X_{n}^{\lambda}$ on 
$\left(\mathbb{R}^{n+1}, \mathcal{B}\left(\mathbb{R}^{n+1}\right)\right)$. For $y \in C[0, t]$, let $K_{F}^{\lambda}\left(y, \vec{\xi}_{n}\right)$ be given by $(1.5)$ where 0 is replaced by $y$. If $I_{F}^{\lambda}\left(0, \vec{\xi}_{n+1}\right)$ has the analytic extension $J_{\lambda}^{*}(F)\left(\vec{\xi}_{n+1}\right)$ on $\mathbb{C}_{+}$as a function of $\lambda$, then it is called the conditional analytic Wiener $w_{\varphi}$-integral of $F$ given $X_{n+1}$ with parameter $\lambda$ and denoted by $E^{a n w_{\lambda}}\left[F \mid X_{n+1}\right]\left(\vec{\xi}_{n+1}\right)=J_{\lambda}^{*}(F)\left(\vec{\xi}_{n+1}\right)$ for $\vec{\xi}_{n+1} \in \mathbb{R}^{n+2}$. Moreover, if for a nonzero real $q, E^{a n w_{\lambda}}\left[F \mid X_{n+1}\right]\left(\vec{\xi}_{n+1}\right)$ has a limit as $\lambda$ approaches to $-i q$ through $\mathbb{C}_{+}$, then it is called the conditional analytic Feynman $w_{\varphi}$-integral of $F$ given $X_{n+1}$ with parameter $q$ and denoted by $E^{a n f_{q}}\left[F \mid X_{n+1}\right]\left(\vec{\xi}_{n+1}\right)=\lim _{\lambda \rightarrow-i q} E^{a n w_{\lambda}}\left[F \mid X_{n+1}\right]\left(\vec{\xi}_{n+1}\right)$. Similarly, the definitions of $E^{a n w_{\lambda}}\left[F \mid X_{n}\right]\left(\vec{\xi}_{n}\right)$ and $E^{a n f_{q}}\left[F \mid X_{n}\right]\left(\vec{\xi}_{n}\right)$ are understood with $K_{F}^{\lambda}\left(0, \vec{\xi}_{n}\right)$ if $X_{n+1}$ is replaced by $X_{n}$.

For a given extended real number $p$ with $1<p \leq \infty$, suppose that $p$ and $p^{\prime}$ are related by $\frac{1}{p}+\frac{1}{p^{\prime}}=1$ (possibly $p^{\prime}=1$ if $\left.p=\infty\right)$. Let $F_{n}$ and $F$ be measurable functions such that $\lim _{n \rightarrow \infty} \int_{C}\left|F_{n}(x)-F(x)\right|^{p^{\prime}} d w_{\varphi}(x)=$ 0 . Then we write $\operatorname{lil.m}_{\cdot n \rightarrow \infty}\left(w^{p^{\prime}}\right)\left(F_{n}\right)=F$ and call $F$ the limit in the mean of order $p^{\prime}$. A similar definition is understood when $n$ is replaced by a continuously varying parameter.

Let $F$ and $G$ be defined on $C[0, t]$ and let $X_{n+1}$ be given by (1.3). For $\lambda \in \mathbb{C}_{+}$and $w_{\varphi}$-a.e. $y \in C[0, t]$, let

$$
T_{\lambda}\left[F \mid X_{n+1}\right]\left(y, \vec{\xi}_{n+1}\right)=E^{a n w_{\lambda}}\left[F(y+\cdot) \mid X_{n+1}\right]\left(\vec{\xi}_{n+1}\right)
$$

for $P_{X_{n+1}}$-a.e. $\vec{\xi}_{n+1} \in \mathbb{R}^{n+2}$ if it exists. For a nonzero real $q$ and $w_{\varphi^{-}}$ a.e. $y \in C[0, t]$, define the $L_{1}$-analytic conditional Fourier-Feynman transform $T_{q}^{(1)}\left[F \mid X_{n+1}\right]$ of $F$ given $X_{n+1}$ by the formula

$$
T_{q}^{(1)}\left[F \mid X_{n+1}\right]\left(y, \vec{\xi}_{n+1}\right)=E^{a n f_{q}}\left[F(y+\cdot) \mid X_{n+1}\right]\left(\vec{\xi}_{n+1}\right)
$$

for $P_{X_{n+1}}$-a.e. $\vec{\xi}_{n+1} \in \mathbb{R}^{n+2}$ if it exists. For $1<p \leq \infty$, define the $L_{p^{-}}$analytic conditional Fourier-Feynman transform $T_{q}^{(p)}\left[F \mid X_{n+1}\right]$ of $F$ given $X_{n+1}$ by the formula

$$
T_{q}^{(p)}\left[F \mid X_{n+1}\right]\left(\cdot, \vec{\xi}_{n+1}\right)=\underset{\lambda \rightarrow-i q}{\operatorname{li.m.}}\left(w^{p^{\prime}}\right)\left(T_{\lambda}\left[F \mid X_{n+1}\right]\left(\cdot, \vec{\xi}_{n+1}\right)\right)
$$

for $P_{X_{n+1}}$-a.e. $\vec{\xi}_{n+1} \in \mathbb{R}^{n+2}$, where $\lambda$ approaches to $-i q$ through $\mathbb{C}_{+}$. We also define the conditional convolution product $\left[(F * G)_{\lambda} \mid X_{n+1}\right]$ of $F$ and $G$ given $X_{n+1}$ by the formula, for $w_{\varphi}$-a.e. $y \in C[0, t]$ 


$$
\begin{aligned}
& {\left[(F * G)_{\lambda} \mid X_{n+1}\right]\left(y, \vec{\xi}_{n+1}\right)} \\
& = \begin{cases}E^{a n w_{\lambda}}\left[F\left(\frac{y+\cdot}{\sqrt{2}}\right) G\left(\frac{y-\cdot}{\sqrt{2}}\right) \mid X_{n+1}\right]\left(\vec{\xi}_{n+1}\right), & \lambda \in \mathbb{C}_{+} ; \\
E^{a n f_{q}}\left[F\left(\frac{y+\cdot}{\sqrt{2}}\right) G\left(\frac{y-\cdot}{\sqrt{2}}\right) \mid X_{n+1}\right]\left(\vec{\xi}_{n+1}\right), & \lambda=-i q\end{cases}
\end{aligned}
$$

if they exist for $P_{X_{n+1}}$-a.e. $\vec{\xi}_{n+1} \in \mathbb{R}^{n+2}$. If $\lambda=-i q$, we replace $[(F *$ $\left.G)_{\lambda} \mid X_{n+1}\right]$ by $\left[(F * G)_{q} \mid X_{n+1}\right]$. Similar definitions and notations are understood with $\vec{\xi}_{n} \in \mathbb{R}^{n+1}$ if $X_{n+1}$ is replaced by $X_{n}$ which is given by (1.4).

\section{Conditional Fourier-Feynman transform with final time conditioning function}

For $v$ in $L_{2}[0, t]$ and $x$ in $C[0, t]$, let $(v, x)$ denote the Paley-WienerZygmund integral of $v$ according to $x([11])$. Note that $\langle\cdot, \cdot\rangle$ and $\langle\cdot, \cdot\rangle_{\mathbb{R}^{r}}$ denote the inner product over $L_{2}[0, t]$ and the dot product on the $r$ dimensional Euclidean space $\mathbb{R}^{r}$, respectively.

For each $j=1, \cdots, n+1$, let $\alpha_{j}=\left(t_{j}-t_{j-1}\right)^{-\frac{1}{2}} \chi_{\left(t_{j-1}, t_{j}\right]}$ on $[0, t]$. Let $V$ be the subspace of $L_{2}[0, t]$ generated by $\left\{\alpha_{1}, \cdots, \alpha_{n+1}\right\}$ and $V^{\perp}$ denote the orthogonal complement of $V$. Let $\mathcal{P}$ and $\mathcal{P}^{\perp}$ be the orthogonal projections from $L_{2}[0, t]$ to $V$ and $V^{\perp}$, respectively. Throughout this paper, let $\left\{v_{1}, v_{2}, \cdots, v_{r}\right\}$ be an orthonormal subset of $L_{2}[0, t]$ such that $\left\{\mathcal{P}^{\perp} v_{1}, \cdots, \mathcal{P}^{\perp} v_{r}\right\}$ is an independent set. Let $\left\{e_{1}, \cdots, e_{r}\right\}$ be the orthonormal set obtained from $\left\{\mathcal{P}^{\perp} v_{1}, \cdots, \mathcal{P}^{\perp} v_{r}\right\}$ by the GramSchmidt orthonormalization process. Now, for $l=1, \cdots, r$, let $\mathcal{P}^{\perp} v_{l}=$ $\sum_{j=1}^{r} \beta_{l j} e_{j}$ be the linear combinations of the $e_{j}$ s and let $A=\left[\beta_{l j}\right]_{r \times r}$ be the coefficient matrix of the combinations. We can regard $A$ as the linear transformation $T_{A}: \mathbb{R}^{r} \rightarrow \mathbb{R}^{r}$ given by $T_{A} \vec{z}=\vec{z} A$, where $\vec{z}$ is any row-vector in $\mathbb{R}^{r}$. Note that $A$ is invertible so that $T_{A}$ is an isomorphism. For $v \in L_{2}[0, t]$, let

$$
c_{j}(v)=\left\langle v, e_{j}\right\rangle
$$

for $j=1, \cdots, r$ and let

$$
(\vec{v}, x)=\left(\left(v_{1}, x\right), \cdots,\left(v_{r}, x\right)\right)
$$

for $x \in C[0, t]$. Furthermore, for $\vec{z} \in \mathbb{R}^{r}$ and $\vec{\xi}_{n+1} \in \mathbb{R}^{n+2}$ let

$$
H_{1}\left(x, \vec{\xi}_{n+1}, v, \vec{z}\right)=\exp \left\{i\left[\left(v, x+\left[\vec{\xi}_{n+1}\right]\right)+\left\langle\left(\vec{v}, x+\left[\vec{\xi}_{n+1}\right]\right), \vec{z}\right\rangle_{\mathbb{R}^{r}}\right]\right\}
$$


and for $\lambda \in \mathbb{C}_{+}^{\sim}$ let

$$
\begin{aligned}
& H_{2}(\lambda, v, \vec{z}) \\
& =\exp \left\{-\frac{1}{2 \lambda}\left[\left\|\mathcal{P}^{\perp} v\right\|_{2}^{2}+2\left\langle\vec{c}\left(\mathcal{P}^{\perp} v\right), T_{A} \vec{z}\right\rangle_{\mathbb{R}^{r}}+\left\|T_{A} \vec{z}\right\|_{\mathbb{R}^{r}}^{2}\right]\right\},
\end{aligned}
$$

where $\vec{c}\left(\mathcal{P}^{\perp} v\right)=\left(c_{1}\left(\mathcal{P}^{\perp} v\right), \cdots, c_{r}\left(\mathcal{P}^{\perp} v\right)\right)$ and the $c_{j}$ s are given by $(2.1)$. Note that by the Bessel's inequality,

$$
\begin{aligned}
& \left|H_{2}(\lambda, v, \vec{z})\right| \\
& =\exp \left\{-\frac{\operatorname{Re} \lambda}{2|\lambda|^{2}}\left[\left\|\mathcal{P}^{\perp} v\right\|_{2}^{2}-\left\|\vec{c}\left(\mathcal{P}^{\perp} v\right)\right\|_{\mathbb{R}^{r}}^{2}+\left\|\vec{c}\left(\mathcal{P}^{\perp} v\right)+T_{A} \vec{z}\right\|_{\mathbb{R}^{r}}^{2}\right]\right\} \\
& \leq 1 .
\end{aligned}
$$

Using the same method as used in the proof of Theorem 2.6 in [10], we can prove the following lemma.

Lemma 2.1. For $x \in C[0, t], \lambda>0, v \in L_{2}[0, t]$ and $\vec{z} \in \mathbb{R}^{r}$, let

$$
H_{3}(\lambda, v, \vec{z}, x)=\exp \left\{i \lambda^{-\frac{1}{2}}\left[(v, x-[x])+\langle(\vec{v}, x-[x]), \vec{z}\rangle_{\mathbb{R}^{r}}\right]\right\} .
$$

Then

$$
\int_{C} H_{3}(\lambda, v, \vec{z}, x) d w_{\varphi}(x)=H_{2}(\lambda, v, \vec{z}),
$$

where $\mathrm{H}_{2}$ is given by (2.4).

Let $\hat{\mathrm{M}}\left(\mathbb{R}^{r}\right)$ be the set of all functions $\phi$ on $\mathbb{R}^{r}$ defined by

$$
\phi(\vec{u})=\int_{\mathbb{R}^{r}} \exp \left\{i\langle\vec{u}, \vec{z}\rangle_{\mathbb{R}^{r}}\right\} d \rho(\vec{z}),
$$

where $\rho$ is a complex Borel measure of bounded variation over $\mathbb{R}^{r}$, and let $\Phi$ be given by

$$
\Phi(x)=\phi(\vec{v}, x)
$$

for $w_{\varphi}$-a.e. $x \in C[0, t]$, where $(\vec{v}, x)$ and $\phi \in \hat{\mathrm{M}}\left(\mathbb{R}^{r}\right)$ are given by $(2.2)$ and (2.7), respectively. Let $\mathcal{M}=\mathcal{M}\left(L_{2}[0, t]\right)$ be the class of all $\mathbb{C}$ valued Borel measures of bounded variation over $L_{2}[0, t]$ and let $\mathcal{S}_{w_{\varphi}}$ be the space of all functions $F$ which for $\sigma \in \mathcal{M}$ have the form

$$
F(x)=\int_{L_{2}[0, t]} \exp \{i(v, x)\} d \sigma(v)
$$

for $w_{\varphi}$-a.e. $x \in C[0, t]$. Note that $\mathcal{S}_{w_{\varphi}}$ is a Banach algebra which is equivalent to $\mathcal{M}$ with the norm $\|F\|=\|\sigma\|$, the total variation of $\sigma$ [11].

Now we have the following theorem. 
Theorem 2.2. Let $1 \leq p \leq \infty$ and $X_{n+1}$ be given by (1.3). For $w_{\varphi^{-}}$ a.e. $x \in C[0, t]$, let $\Psi(x)=F(x) \Phi(x)$, where $\Phi$ and $F$ are given by (2.8) and (2.9), respectively. Then for a nonzero real $q$, $w_{\varphi}$-a.e. $y \in C[0, t]$ and $P_{X_{n+1}}$-a.e. $\vec{\xi}_{n+1} \in \mathbb{R}^{n+2}$,

$$
\begin{aligned}
& T_{q}^{(p)}\left[\Psi \mid X_{n+1}\right]\left(y, \vec{\xi}_{n+1}\right) \\
& =\int_{\mathbb{R}^{r}} \int_{L_{2}[0, t]} H_{1}\left(y, \vec{\xi}_{n+1}, v, \vec{z}\right) H_{2}(-i q, v, \vec{z}) d \sigma(v) d \rho(\vec{z}),
\end{aligned}
$$

where $H_{1}$ and $H_{2}$ are given by (2.3) and (2.4), respectively.

Proof. For $\lambda>0, y \in C[0, t]$ and $\vec{\xi}_{n+1} \in \mathbb{R}^{n+2}$,

$$
\begin{aligned}
& I_{\Psi}^{\lambda}\left(y, \vec{\xi}_{n+1}\right) \\
& =\int_{\mathbb{R}^{r}} \int_{L_{2}[0, t]} \int_{C} H_{1}\left(y, \vec{\xi}_{n+1}, v, \vec{z}\right) H_{3}(\lambda, v, \vec{z}, x) d w_{\varphi}(x) d \sigma(v) d \rho(\vec{z}),
\end{aligned}
$$

where $H_{3}$ is given by (2.6). By Lemma 2.1, we obtain that

$$
I_{\Psi}^{\lambda}\left(y, \vec{\xi}_{n+1}\right)=\int_{\mathbb{R}^{r}} \int_{L_{2}[0, t]} H_{1}\left(y, \vec{\xi}_{n+1}, v, \vec{z}\right) H_{2}(\lambda, v, \vec{z}) d \sigma(v) d \rho(\vec{z}) .
$$

By (2.5), the Moreras theorem and the dominated convergence theorem, we have the existence of $T_{\lambda}\left[\Psi \mid X_{n+1}\right]\left(y, \vec{\xi}_{n+1}\right)$ as the analytic extension of $I_{\Psi}^{\lambda}\left(y, \vec{\xi}_{n+1}\right)$ on $\mathbb{C}_{+}$. Let $T_{q}^{(p)}\left[\Psi \mid X_{n+1}\right]\left(y, \vec{\xi}_{n+1}\right)$ be given by $(2.10)$ and $\frac{1}{p}+\frac{1}{p^{\prime}}=1$. Then

$$
\begin{aligned}
& \left\|T_{\lambda}\left[\Psi \mid X_{n+1}\right]\left(\cdot, \vec{\xi}_{n+1}\right)-T_{q}^{(p)}\left[\Psi \mid X_{n+1}\right]\left(\cdot, \vec{\xi}_{n+1}\right)\right\|_{p^{\prime}} \\
& \leq \int_{\mathbb{R}^{r}} \int_{L_{2}[0, t]}\left|H_{2}(\lambda, v, \vec{z})-H_{2}(-i q, v, \vec{z})\right| d|\sigma|(v) d|\rho|(\vec{z})
\end{aligned}
$$

which converges to 0 as $\lambda$ approaches to $-i q$ through $\mathbb{C}_{+}$by the dominated convergence theorem. Now, the proof is completed.

TheOREM 2.3. Let $\phi_{1}, \phi_{2}$ and $\rho_{1}, \rho_{2}$ be related by (2.7), respectively, and let $F_{1}, F_{2}$ and $\sigma_{1}, \sigma_{2}$ be related by (2.9), respectively. Let $\Psi_{1}(x)=$ $F_{1}(x) \phi_{1}(\vec{v}, x)$ and $\Psi_{2}(x)=F_{2}(x) \phi_{2}(\vec{v}, x)$ for $w_{\varphi}$-a.e. $x \in C[0, t]$. Furthermore, let $X_{n+1}$ be given by (1.3). Then for a nonzero real $q, w_{\varphi}$-a.e. 
$y \in C[0, t]$ and $P_{X_{n+1}}$-a.e. $\vec{\xi}_{n+1} \in \mathbb{R}^{n+2}$,

$$
\begin{aligned}
& {\left[\left(\Psi_{1} * \Psi_{2}\right)_{q} \mid X_{n+1}\right]\left(y, \vec{\xi}_{n+1}\right)} \\
& =\int_{\mathbb{R}^{r}} \int_{\mathbb{R}^{r}} \int_{L_{2}[0, t]} \int_{L_{2}[0, t]} H_{1}\left(y, \vec{\xi}_{n+1}, \frac{1}{\sqrt{2}} v_{1}, \frac{1}{\sqrt{2}} \vec{z}_{1}\right) H_{1}(y, \\
& \left.-\vec{\xi}_{n+1}, \frac{1}{\sqrt{2}} v_{2}, \frac{1}{\sqrt{2}} \vec{z}_{2}\right) H_{2}\left(-i q, \frac{1}{\sqrt{2}}\left(v_{1}-v_{2}\right), \frac{1}{\sqrt{2}}\left(\vec{z}_{1}-\vec{z}_{2}\right)\right) \\
& d \sigma_{1}\left(v_{1}\right) d \sigma_{2}\left(v_{2}\right) d \rho_{1}\left(\vec{z}_{1}\right) d \rho_{2}\left(\vec{z}_{2}\right),
\end{aligned}
$$

where $H_{1}$ and $H_{2}$ are given by (2.3) and (2.4), respectively.

Proof. For $\lambda>0, w_{\varphi}$-a.e. $y \in C[0, t]$ and $P_{X_{n+1}}$-a.e. $\vec{\xi}_{n+1} \in \mathbb{R}^{n+2}$,

$$
\begin{aligned}
& {\left[\left(\Psi_{1} * \Psi_{2}\right)_{\lambda} \mid X_{n+1}\right]\left(y, \vec{\xi}_{n+1}\right)} \\
& =\int_{\mathbb{R}^{r}} \int_{\mathbb{R}^{r}} \int_{L_{2}[0, t]} \int_{L_{2}[0, t]} \int_{C} \exp \left\{\frac { i } { \sqrt { 2 } } \left[\left(v_{1}, y+\left[\vec{\xi}_{n+1}\right]\right)+\langle(\vec{v}, y+\right.\right. \\
& \left.\left.\left.\left[\vec{\xi}_{n+1}\right]\right), \vec{z}_{1}\right\rangle_{\mathbb{R}^{r}}+\left(v_{2}, y-\left[\vec{\xi}_{n+1}\right]\right)+\left\langle\left(\vec{v}, y-\left[\vec{\xi}_{n+1}\right]\right), \vec{z}_{2}\right\rangle_{\mathbb{R}^{r}}\right]+ \\
& \left.\frac{i}{\sqrt{2 \lambda}}\left[\left(v_{1}-v_{2}, x-[x]\right)+\left\langle(\vec{v}, x-[x]), \vec{z}_{1}-\vec{z}_{2}\right\rangle_{\mathbb{R}^{r}}\right]\right\} d w_{\varphi}(x) \\
& d \sigma_{1}\left(v_{1}\right) d \sigma_{2}\left(v_{2}\right) d \rho_{1}\left(\vec{z}_{1}\right) d \rho_{2}\left(\vec{z}_{2}\right) \\
& =\int_{\mathbb{R}^{r}} \int_{\mathbb{R}^{r}} \int_{L_{2}[0, t]} \int_{L_{2}[0, t]} \int_{C} H_{1}\left(y, \vec{\xi}_{n+1}, \frac{1}{\sqrt{2}} v_{1}, \frac{1}{\sqrt{2}} \vec{z}_{1}\right) H_{1}(y, \\
& \left.-\vec{\xi}_{n+1}, \frac{1}{\sqrt{2}} v_{2}, \frac{1}{\sqrt{2}} \vec{z}_{2}\right) H_{3}\left(\lambda, \frac{1}{\sqrt{2}}\left(v_{1}-v_{2}\right), \frac{1}{\sqrt{2}}\left(\vec{z}_{1}-\vec{z}_{2}\right), x\right) \\
& d w_{\varphi}(x) d \sigma_{1}\left(v_{1}\right) d \sigma_{2}\left(v_{2}\right) d \rho_{1}\left(\vec{z}_{1}\right) d \rho_{2}\left(\vec{z}_{2}\right),
\end{aligned}
$$

where $H_{3}$ is given by (2.6). By Lemma 2.1, we obtain that

$$
\begin{aligned}
& {\left[\left(\Psi_{1} * \Psi_{2}\right)_{\lambda} \mid X_{n+1}\right]\left(y, \vec{\xi}_{n+1}\right)} \\
& =\int_{\mathbb{R}^{r}} \int_{\mathbb{R}^{r}} \int_{L_{2}[0, t]} \int_{L_{2}[0, t]} H_{1}\left(y, \vec{\xi}_{n+1}, \frac{1}{\sqrt{2}} v_{1}, \frac{1}{\sqrt{2}} \vec{z}_{1}\right) H_{1}(y, \\
& \left.-\vec{\xi}_{n+1}, \frac{1}{\sqrt{2}} v_{2}, \frac{1}{\sqrt{2}} \vec{z}_{2}\right) H_{2}\left(\lambda, \frac{1}{\sqrt{2}}\left(v_{1}-v_{2}\right), \frac{1}{\sqrt{2}}\left(\vec{z}_{1}-\vec{z}_{2}\right)\right) \\
& d \sigma_{1}\left(v_{1}\right) d \sigma_{2}\left(v_{2}\right) d \rho_{1}\left(\vec{z}_{1}\right) d \rho_{2}\left(\vec{z}_{2}\right) .
\end{aligned}
$$

By (2.5), the Morera's theorem and the dominated convergence theorem, we have the result. 


\section{Conditional Fourier-Feynman transform without final time conditioning function}

In this section, we evaluate time-independent conditional FourierFeynman transform and conditional convolution product of the functions as given in the previous section.

Lemma 3.1. For $\vec{\xi}_{n}=\left(\xi_{0}, \xi_{1}, \cdots, \xi_{n}\right) \in \mathbb{R}^{n+1}$, let $\vec{\xi}_{n+1}=\left(\xi_{0}, \xi_{1}, \cdots\right.$, $\left.\xi_{n}, \xi_{n+1}\right)$, where $\xi_{n+1} \in \mathbb{R}$. Furthermore, for $y \in C[0, t], v \in L_{2}[0, t]$, $\vec{z}=\left(z_{1}, \cdots, z_{r}\right) \in \mathbb{R}^{r}$, let $H_{1}\left(y, \vec{\xi}_{n+1}, v, \vec{z}\right)$ be given by $(2.3)$, let

$$
H_{4}\left(\vec{\xi}_{n}, v, \vec{z}\right)=\exp \left\{i \sum_{j=1}^{n}\left(\xi_{j}-\xi_{j-1}\right)\left[(\mathcal{P} v)\left(t_{j}\right)+\sum_{l=1}^{r} z_{l}\left(\mathcal{P} v_{l}\right)\left(t_{j}\right)\right]\right\}
$$

and for $\lambda \in \mathbb{C}_{+}^{\sim}$, let

$$
H_{5}(\lambda, v, \vec{z})=\exp \left\{-\frac{1}{2 \lambda}\left[(\mathcal{P} v)(t)+\sum_{l=1}^{r} z_{l}\left(\mathcal{P} v_{l}\right)(t)\right]^{2}\right\} .
$$

Then for $\lambda>0$,

$$
\begin{aligned}
& {\left[\frac{\lambda}{2 \pi\left(t-t_{n}\right)}\right]^{\frac{1}{2}} \int_{\mathbb{R}} H_{1}\left(y, \vec{\xi}_{n+1}, v, \vec{z}\right) \exp \left\{-\frac{\lambda\left(\xi_{n+1}-\xi_{n}\right)^{2}}{2\left(t-t_{n}\right)}\right\} d \xi_{n+1}} \\
& =H_{1}(y, 0, v, \vec{z}) H_{4}\left(\vec{\xi}_{n}, v, \vec{z}\right) H_{5}(\lambda, v, \vec{z}) .
\end{aligned}
$$

Proof. By the definition of the Paley-Wiener-Zygmund integral, it is not difficult to show that for $v \in L_{2}[0, t]$

$$
\left(v,\left[\vec{\xi}_{n+1}\right]\right)=\sum_{j=1}^{n}(\mathcal{P} v)\left(t_{j}\right)\left(\xi_{j}-\xi_{j-1}\right)+(\mathcal{P} v)(t)\left(\xi_{n+1}-\xi_{n}\right) .
$$

Now, let $I_{\lambda}$ be the left hand side of (3.3). Then

$$
\begin{aligned}
I_{\lambda}= & H_{1}(y, 0, v, \vec{z})\left[\frac{\lambda}{2 \pi\left(t-t_{n}\right)}\right]^{\frac{1}{2}} \int_{\mathbb{R}} H_{4}\left(\vec{\xi}_{n}, v, \vec{z}\right) \exp \{i[(\mathcal{P} v)(t) \\
& \left.\left.+\sum_{l=1}^{r} z_{l}\left(\mathcal{P} v_{l}\right)(t)\right]\left(\xi_{n+1}-\xi_{n}\right)-\frac{\lambda\left(\xi_{n+1}-\xi_{n}\right)^{2}}{2\left(t-t_{n}\right)}\right\} d \xi_{n+1} \\
= & H_{1}(y, 0, v, \vec{z}) H_{4}\left(\vec{\xi}_{n}, v, \vec{z}\right) H_{5}(\lambda, v, \vec{z})
\end{aligned}
$$

by the following well-known integration formula

$$
\int_{\mathbb{R}} \exp \left\{-a u^{2}+i b u\right\} d u=\left(\frac{\pi}{a}\right)^{\frac{1}{2}} \exp \left\{-\frac{b^{2}}{4 a}\right\}
$$


for $a \in \mathbb{C}_{+}$and $b \in \mathbb{R}$. Now, the proof is completed.

Theorem 3.2. Let $1 \leq p \leq \infty$ and $X_{n}$ be given by (1.4). For $w_{\varphi}$-a.e. $x \in C[0, t]$, let $\Psi(x)=F(x) \Phi(x)$, where $\Phi$ and $F$ are given by (2.8) and (2.9), respectively. Then for a nonzero real $q, w_{\varphi}$-a.e. $y \in C[0, t]$ and $P_{X_{n}}$-a.e. $\vec{\xi}_{n} \in \mathbb{R}^{n+1}$,

$$
\begin{aligned}
T_{q}^{(p)}\left[\Psi \mid X_{n}\right]\left(y, \vec{\xi}_{n}\right)= & \int_{\mathbb{R}^{r}} \int_{L_{2}[0, t]} H_{1}(y, 0, v, \vec{z}) H_{4}\left(\vec{\xi}_{n}, v, \vec{z}\right) \\
& \times H_{2}(-i q, v, \vec{z}) H_{5}(-i q, v, \vec{z}) d \sigma(v) d \rho(\vec{z}),
\end{aligned}
$$

where $H_{1}, H_{2}, H_{4}$, and $H_{5}$ are given by (2.3), (2.4), (3.1) and (3.2), respectively.

Proof. For $\vec{\xi}_{n}=\left(\xi_{0}, \xi_{1}, \cdots, \xi_{n}\right) \in \mathbb{R}^{n+1}$, let $\vec{\xi}_{n+1}=\left(\xi_{0}, \xi_{1}, \cdots, \xi_{n}\right.$, $\left.\xi_{n+1}\right)$, where $\xi_{n+1} \in \mathbb{R}$. For $\lambda>0, y \in C[0, t]$ and $\vec{\xi}_{n} \in \mathbb{R}^{n+1}$,

$$
\begin{aligned}
K_{\Psi}^{\lambda}\left(y, \vec{\xi}_{n}\right)= & {\left[\frac{\lambda}{2 \pi\left(t-t_{n}\right)}\right]^{\frac{1}{2}} \int_{\mathbb{R}} I_{\Psi}^{\lambda}\left(y, \vec{\xi}_{n+1}\right) \exp \left\{-\frac{\lambda\left(\xi_{n+1}-\xi_{n}\right)^{2}}{2\left(t-t_{n}\right)}\right\} d \xi_{n+1} } \\
= & {\left[\frac{\lambda}{2 \pi\left(t-t_{n}\right)}\right]^{\frac{1}{2}} \int_{\mathbb{R}^{r}} \int_{L_{2}[0, t]} H_{2}(\lambda, v, \vec{z}) \int_{\mathbb{R}} H_{1}\left(y, \vec{\xi}_{n+1}, v, \vec{z}\right) } \\
& \times \exp \left\{-\frac{\lambda\left(\xi_{n+1}-\xi_{n}\right)^{2}}{2\left(t-t_{n}\right)}\right\} d \xi_{n+1} d \sigma(v) d \rho(\vec{z})
\end{aligned}
$$

by Theorem 2.2. By Lemma 3.1, we obtain that

$$
\begin{aligned}
K_{\Psi}^{\lambda}\left(y, \vec{\xi}_{n}\right)= & \int_{\mathbb{R}^{r}} \int_{L_{2}[0, t]} H_{1}(y, 0, v, \vec{z}) H_{2}(\lambda, v, \vec{z}) H_{4}\left(\vec{\xi}_{n}, v, \vec{z}\right) \\
& \times H_{5}(\lambda, v, \vec{z}) d \sigma(v) d \rho(\vec{z}) .
\end{aligned}
$$

By (2.5), the Morera's theorem and the dominated convergence theorem, we have the existence of $T_{\lambda}\left[\Psi \mid X_{n}\right]\left(y, \vec{\xi}_{n}\right)$ as the analytic extension of $K_{\Psi}^{\lambda}\left(y, \vec{\xi}_{n}\right)$ on $\mathbb{C}_{+}$. Let $\frac{1}{p}+\frac{1}{p^{\prime}}=1$. Then for $\lambda \in \mathbb{C}_{+}$,

$$
\begin{aligned}
& \left\|T_{\lambda}\left[\Psi \mid X_{n}\right]\left(\cdot, \vec{\xi}_{n}\right)-T_{q}^{(p)}\left[\Psi \mid X_{n}\right]\left(\cdot, \vec{\xi}_{n}\right)\right\|_{p^{\prime}} \\
& \leq \int_{\mathbb{R}^{r}} \int_{L_{2}[0, t]}\left|H_{2}(\lambda, v, \vec{z}) H_{5}(\lambda, v, \vec{z})-H_{2}(-i q, v, \vec{z}) H_{5}(-i q, v, \vec{z})\right| d|\sigma|(v) d|\rho|(\vec{z})
\end{aligned}
$$

which converges to 0 as $\lambda$ approaches to $-i q$ through $\mathbb{C}_{+}$. Now, the proof is completed. 
Theorem 3.3. Let $\Psi_{1}$ and $\Psi_{2}$ be as given in Theorem 2.3, and let $X_{n}$ be given by (1.4). Then for a nonzero real $q, w_{\varphi}$-a.e. $y \in C[0, t]$ and $P_{X_{n}}$-a.e. $\vec{\xi}_{n} \in \mathbb{R}^{n+1},\left[\left(\Psi_{1} * \Psi_{2}\right)_{q} \mid X_{n}\right]\left(y, \vec{\xi}_{n}\right)$ is given by

$$
\begin{aligned}
& {\left[\left(\Psi_{1} * \Psi_{2}\right)_{q} \mid X_{n}\right]\left(y, \vec{\xi}_{n}\right)} \\
& =\int_{\mathbb{R}^{r}} \int_{\mathbb{R}^{r}} \int_{L_{2}[0, t]} \int_{L_{2}[0, t]} H_{1}\left(y, 0, \frac{1}{\sqrt{2}}\left(v_{1}+v_{2}\right), \frac{1}{\sqrt{2}}\left(\vec{z}_{1}+\vec{z}_{2}\right)\right) H_{4}\left(\vec{\xi}_{n},\right. \\
& \left.\frac{1}{\sqrt{2}}\left(v_{1}-v_{2}\right), \frac{1}{\sqrt{2}}\left(\vec{z}_{1}-\vec{z}_{2}\right)\right) H_{2}\left(-i q, \frac{1}{\sqrt{2}}\left(v_{1}-v_{2}\right), \frac{1}{\sqrt{2}}\left(\vec{z}_{1}-\vec{z}_{2}\right)\right) \\
& \times H_{5}\left(-i q, \frac{1}{\sqrt{2}}\left(v_{1}-v_{2}\right), \frac{1}{\sqrt{2}}\left(\vec{z}_{1}-\vec{z}_{2}\right)\right) d \sigma_{1}\left(v_{1}\right) d \sigma_{2}\left(v_{2}\right) d \rho_{1}\left(\vec{z}_{1}\right) d \rho_{2}\left(\vec{z}_{2}\right),
\end{aligned}
$$

where $H_{1}, H_{2}, H_{4}$ and $H_{5}$ are given by (2.3), (2.4), (3.1) and (3.2), respectively.

Proof. For $\vec{\xi}_{n}=\left(\xi_{0}, \xi_{1}, \cdots, \xi_{n}\right) \in \mathbb{R}^{n+1}$, let $\vec{\xi}_{n+1}=\left(\xi_{0}, \xi_{1}, \cdots, \xi_{n}\right.$, $\left.\xi_{n+1}\right)$, where $\xi_{n+1} \in \mathbb{R}$. Note that for $y \in C[0, t]$, for $v_{1}, v_{2} \in L_{2}[0, t]$ and for $\vec{z}_{1}, \vec{z}_{2} \in \mathbb{R}^{r}$

$$
\begin{aligned}
& H_{1}\left(y, \vec{\xi}_{n+1}, \frac{1}{\sqrt{2}} v_{1}, \frac{1}{\sqrt{2}} \vec{z}_{1}\right) H_{1}\left(y,-\vec{\xi}_{n+1}, \frac{1}{\sqrt{2}} v_{2}, \frac{1}{\sqrt{2}} \vec{z}_{2}\right) \\
& =H_{1}\left(y, 0, \frac{1}{\sqrt{2}}\left(v_{1}+v_{2}\right), \frac{1}{\sqrt{2}}\left(\vec{z}_{1}+\vec{z}_{2}\right)\right) \\
& \quad \times H_{1}\left(0, \vec{\xi}_{n+1}, \frac{1}{\sqrt{2}}\left(v_{1}-v_{2}\right), \frac{1}{\sqrt{2}}\left(\vec{z}_{1}-\vec{z}_{2}\right)\right)
\end{aligned}
$$

so that we have for $\lambda>0$

$$
\begin{aligned}
I_{\lambda}\left(\vec{\xi}_{n}\right) \equiv & {\left[\frac{\lambda}{2 \pi\left(t-t_{n}\right)}\right]^{\frac{1}{2}} \int_{\mathbb{R}} H_{1}\left(y, \vec{\xi}_{n+1}, \frac{1}{\sqrt{2}} v_{1}, \frac{1}{\sqrt{2}} \vec{z}_{1}\right) H_{1}\left(y,-\vec{\xi}_{n+1},\right.} \\
& \left.\frac{1}{\sqrt{2}} v_{2}, \frac{1}{\sqrt{2}} \vec{z}_{2}\right) \exp \left\{-\frac{\lambda\left(\xi_{n+1}-\xi_{n}\right)^{2}}{2\left(t-t_{n}\right)}\right\} d \xi_{n+1} \\
= & H_{1}\left(y, 0, \frac{1}{\sqrt{2}}\left(v_{1}+v_{2}\right), \frac{1}{\sqrt{2}}\left(\vec{z}_{1}+\vec{z}_{2}\right)\right) H_{4}\left(\vec{\xi}_{n}, \frac{1}{\sqrt{2}}\left(v_{1}-v_{2}\right),\right. \\
& \left.\frac{1}{\sqrt{2}}\left(\vec{z}_{1}-\vec{z}_{2}\right)\right) H_{5}\left(\lambda, \frac{1}{\sqrt{2}}\left(v_{1}-v_{2}\right), \frac{1}{\sqrt{2}}\left(\vec{z}_{1}-\vec{z}_{2}\right)\right)
\end{aligned}
$$

by Lemma 3.1. Now, by Theorem 2.3, we obtain that 


$$
\begin{aligned}
& {\left[\left(\Psi_{1} * \Psi_{2}\right)_{\lambda} \mid X_{n}\right]\left(y, \vec{\xi}_{n}\right)} \\
& =\int_{\mathbb{R}^{r}} \int_{\mathbb{R}^{r}} \int_{L_{2}[0, t]} \int_{L_{2}[0, t]} H_{1}\left(y, 0, \frac{1}{\sqrt{2}}\left(v_{1}+v_{2}\right), \frac{1}{\sqrt{2}}\left(\vec{z}_{1}+\vec{z}_{2}\right)\right) H_{2}(\lambda, \\
& \left.\frac{1}{\sqrt{2}}\left(v_{1}-v_{2}\right), \frac{1}{\sqrt{2}}\left(\vec{z}_{1}-\vec{z}_{2}\right)\right) H_{4}\left(\vec{\xi}_{n}, \frac{1}{\sqrt{2}}\left(v_{1}-v_{2}\right), \frac{1}{\sqrt{2}}\left(\vec{z}_{1}-\vec{z}_{2}\right)\right) \\
& \times H_{5}\left(\lambda, \frac{1}{\sqrt{2}}\left(v_{1}-v_{2}\right), \frac{1}{\sqrt{2}}\left(\vec{z}_{1}-\vec{z}_{2}\right)\right) d \sigma_{1}\left(v_{1}\right) d \sigma_{2}\left(v_{2}\right) d \rho_{1}\left(\vec{z}_{1}\right) d \rho_{2}\left(\vec{z}_{2}\right) .
\end{aligned}
$$

By (2.5), the Morera's theorem and the dominated convergence theorem, we have the result.

\section{Relationships between conditional Fourier-Feynman trans- forms and convolutions}

In this section, we investigate the inverse conditional transform of the conditional Fourier-Feynman transforms of the functions as given in the previous sections.

Theorem 4.1. Let $1 \leq p \leq \infty$. Then, under the assumptions as given in Theorem 2.2, we have for $P_{X_{n+1}}$-a.e. $\vec{\xi}_{n+1}, \vec{\zeta}_{n+1} \in \mathbb{R}^{n+2}$

$$
\left\|T_{\bar{\lambda}}\left[T_{\lambda}\left[\Psi \mid X_{n+1}\right]\left(\cdot, \vec{\xi}_{n+1}\right) \mid X_{n+1}\right]\left(\cdot, \vec{\zeta}_{n+1}\right)-\Psi\left(\cdot+\left[\vec{\zeta}_{n+1}+\vec{\xi}_{n+1}\right]\right)\right\|_{p} \rightarrow 0
$$

as $\lambda$ approaches to $-i q$ through $\mathbb{C}_{+}$.

Proof. For $\lambda_{1}>0, \lambda \in \mathbb{C}_{+}, w_{\varphi}$-a.e. $y \in C[0, t]$ and $P_{X_{n+1}}$-a.e. $\vec{\xi}_{n+1}, \vec{\zeta}_{n+1} \in \mathbb{R}^{n+2}$

$$
\begin{aligned}
& I_{T_{\lambda}\left[\Psi \mid X_{n+1}\right]\left(\cdot, \vec{\xi}_{n+1}\right)}^{\lambda_{1}}\left(y, \vec{\zeta}_{n+1}\right) \\
& =\int_{\mathbb{R}^{r}} \int_{L_{2}[0, t]} \int_{C} H_{1}\left(\lambda_{1}^{-\frac{1}{2}}(x-[x])+y+\left[\vec{\zeta}_{n+1}\right], \vec{\xi}_{n+1}, v, \vec{z}\right) H_{2}(\lambda, v, \vec{z}) \\
& \quad d w_{\varphi}(x) d \sigma(v) d \rho(\vec{z}) \\
& =\int_{\mathbb{R}^{r}} \int_{L_{2}[0, t]} \int_{C} H_{1}\left(y, \vec{\zeta}_{n+1}+\vec{\xi}_{n+1}, v, \vec{z}\right) H_{2}(\lambda . v \cdot \vec{z}) H_{3}\left(\lambda_{1}, v, \vec{z}, x\right) \\
& \quad d w_{\varphi}(x) d \sigma(v) d \rho(\vec{z}) \\
& =\int_{\mathbb{R}^{r}} \int_{L_{2}[0, t]} H_{1}\left(y, \vec{\zeta}_{n+1}+\vec{\xi}_{n+1}, v, \vec{z}\right) H_{2}\left(\lambda_{1}, v, \vec{z}\right) H_{2}(\lambda, v, \vec{z}) d \sigma(v) d \rho(\vec{z})
\end{aligned}
$$


by Lemma 2.1 and Theorem 2.2, where $H_{1}, H_{2}$ and $H_{3}$ are given by (2.3), (2.4) and (2.6), respectively. By (2.5) and the Morera's Theorem, we have for $\lambda_{1} \in \mathbb{C}_{+}$

$$
\begin{aligned}
& T_{\lambda_{1}}\left[T_{\lambda}\left[\Psi \mid X_{n+1}\right]\left(\cdot, \vec{\xi}_{n+1}\right) \mid X_{n+1}\right]\left(y, \vec{\zeta}_{n+1}\right) \\
& =\int_{\mathbb{R}^{r}} \int_{L_{2}[0, t]} H_{1}\left(y, \vec{\zeta}_{n+1}+\vec{\xi}_{n+1}, v, \vec{z}\right) H_{2}\left(\lambda_{1}, v, \vec{z}\right) H_{2}(\lambda, v, \vec{z}) d \sigma(v) d \rho(\vec{z}) .
\end{aligned}
$$

Now, we have for $\lambda \in \mathbb{C}_{+}$

$$
\begin{aligned}
& T_{\bar{\lambda}}\left[T_{\lambda}\left[\Psi \mid X_{n+1}\right]\left(\cdot, \vec{\xi}_{n+1}\right) \mid X_{n+1}\right]\left(y, \vec{\zeta}_{n+1}\right) \\
& =\int_{\mathbb{R}^{r}} \int_{L_{2}[0, t]} H_{1}\left(y, \vec{\zeta}_{n+1}+\vec{\xi}_{n+1}, v, \vec{z}\right) H_{2}\left(\frac{|\lambda|^{2}}{2 \operatorname{Re} \lambda}, v, \vec{z}\right) d \sigma(v) d \rho(\vec{z})
\end{aligned}
$$

so that

$$
\begin{aligned}
& \left\|T_{\bar{\lambda}}\left[T_{\lambda}\left[\Psi \mid X_{n+1}\right]\left(\cdot, \vec{\xi}_{n+1}\right) \mid X_{n+1}\right]\left(\cdot, \vec{\zeta}_{n+1}\right)-\Psi\left(\cdot+\left[\vec{\zeta}_{n+1}+\vec{\xi}_{n+1}\right]\right)\right\|_{p} \\
& \leq \int_{\mathbb{R}^{r}} \int_{L_{2}[0, t]}\left[1-H_{2}\left(\frac{|\lambda|^{2}}{2 \operatorname{Re} \lambda}, v, \vec{z}\right)\right] d|\sigma|(v) d|\rho|(\vec{z})
\end{aligned}
$$

which converges to 0 as $\lambda$ approaches to $-i q$ through $\mathbb{C}_{+}$by the dominated convergence theorem.

Theorem 4.2. Let $1 \leq p \leq \infty$ and $X_{n}$ be given by (1.4). For $w_{\varphi}$-a.e. $x \in C[0, t]$, let $\Psi(x)=F(x) \Phi(x)$, where $\Phi$ and $F$ are given by (2.8) and (2.9), respectively. Furthermore, for $y \in C[0, t]$ and $\vec{u}_{n} \in \mathbb{R}^{n+1}$ let

$$
\begin{aligned}
& \Psi_{1}\left(y, \vec{u}_{n}\right) \\
& =\int_{\mathbb{R}^{r}} \int_{L_{2}[0, t]} \exp \{i(v, y)\} \exp \left\{i\langle(\vec{v}, y), \vec{z}\rangle_{\mathbb{R}^{r}}\right\} H_{4}\left(\vec{u}_{n}, v, \vec{z}\right) d \sigma(v) d \rho(\vec{z}),
\end{aligned}
$$

where $H_{4}$ is given by (3.1). Then, for $P_{X_{n}}$-a.e. $\vec{\xi}_{n}, \vec{\zeta}_{n} \in \mathbb{R}^{n+1}$

$$
\left\|T_{\bar{\lambda}}\left[T_{\lambda}\left[\Psi \mid X_{n}\right]\left(\cdot, \vec{\xi}_{n}\right) \mid X_{n}\right]\left(\cdot, \vec{\zeta}_{n}\right)-\Psi_{1}\left(\cdot, \vec{\zeta}_{n}+\vec{\xi}_{n}\right)\right\|_{p} \rightarrow 0
$$

as $\lambda$ approaches to $-i q$ through $\mathbb{C}_{+}$.

Proof. For $\vec{\zeta}_{n}=\left(\zeta_{0}, \zeta_{1}, \cdots, \zeta_{n}\right) \in \mathbb{R}$, let $\vec{\zeta}_{n+1}=\left(\zeta_{0}, \zeta_{1}, \cdots, \zeta_{n}, \zeta_{n+1}\right)$, where $\zeta_{n+1} \in \mathbb{R}$. Then, for $\lambda_{1}>0$ and $\lambda \in \mathbb{C}_{+}$

$$
\begin{aligned}
I_{T_{\lambda}\left[\Psi \mid X_{n}\right]\left(\cdot \vec{\xi}_{n}\right)}^{\lambda_{1}}\left(y, \vec{\zeta}_{n+1}\right)= & \int_{\mathbb{R}^{r}} \int_{L_{2}[0, t]} H_{1}\left(y, \vec{\zeta}_{n+1}, v, \vec{z}\right) H_{2}\left(\lambda_{1}, v, \vec{z}\right) \\
& \times H_{2}(\lambda, v, \vec{z}) H_{4}\left(\vec{\xi}_{n}, v, \vec{z}\right) H_{5}(\lambda, v, \vec{z}) d \sigma(v) d \rho(\vec{z})
\end{aligned}
$$


by Theorems 2.2 and 3.2 , so that by Lemma 3.1

$$
\begin{aligned}
& K_{T_{\lambda}\left[\Psi \mid X_{n}\right]\left(\cdot \vec{\xi}_{n}\right)}^{\lambda_{1}}\left(y, \vec{\zeta}_{n}\right) \\
& =\left[\frac{\lambda}{2 \pi\left(t-t_{n}\right)}\right]^{\frac{1}{2}} \int_{\mathbb{R}} I_{T_{\lambda}\left[\Psi \mid X_{n}\right]\left(\cdot, \vec{\xi}_{n}\right)}^{\lambda_{1}}\left(y, \vec{\zeta}_{n+1}\right) \exp \left\{-\frac{\lambda\left(\zeta_{n+1}-\zeta_{n}\right)^{2}}{2\left(t-t_{n}\right)}\right\} d \zeta_{n+1} \\
& =\int_{\mathbb{R}^{r}} \int_{L_{2}[0, t]} H_{1}(y, 0, v, \vec{z}) H_{2}\left(\lambda_{1}, v, \vec{z}\right) H_{2}(\lambda, v, \vec{z}) H_{4}\left(\vec{\zeta}_{n}+\vec{\xi}_{n}, v, \vec{z}\right) \\
& \quad \times H_{5}\left(\lambda_{1}, v, \vec{z}\right) H_{5}(\lambda, v, \vec{z}) d \sigma(v) d \rho(\vec{z}),
\end{aligned}
$$

where $H_{1}, H_{2}$ and $H_{5}$ are given by (2.3), (2.4) and (3.2), respectively. By (2.5), the Moreras theorem and the dominated convergence theorem, we have the existence of $T_{\lambda_{1}}\left[T_{\lambda}\left[\Psi \mid X_{n}\right]\left(\cdot, \vec{\xi}_{n}\right) \mid X_{n}\right]\left(y, \vec{\zeta}_{n}\right)$ with respect to $\lambda_{1} \in \mathbb{C}_{+}$as the analytic extension of $K_{T_{\lambda}\left[\Psi \mid X_{n}\right]\left(\cdot, \vec{\xi}_{n}\right)}^{\lambda_{1}}\left(y, \vec{\zeta}_{n}\right)$. Now, for $\lambda \in \mathbb{C}_{+}$and $y \in C[0, t]$

$$
\begin{aligned}
& T_{\bar{\lambda}}\left[T_{\lambda}\left[\Psi \mid X_{n}\right]\left(\cdot, \vec{\xi}_{n}\right) \mid X_{n}\right]\left(y, \vec{\zeta}_{n}\right) \\
& =\int_{\mathbb{R}^{r}} \int_{L_{2}[0, t]} H_{1}(y, 0, v, \vec{z}) H_{4}\left(\vec{\zeta}_{n}+\vec{\xi}_{n}, v, \vec{z}\right) H_{2}\left(\frac{|\lambda|^{2}}{2 \operatorname{Re} \lambda}, v, \vec{z}\right) \\
& \quad \times H_{5}\left(\frac{|\lambda|^{2}}{2 \operatorname{Re} \lambda}, v, \vec{z}\right) d \sigma(v) d \rho(\vec{z})
\end{aligned}
$$

so that we have

$$
\begin{aligned}
& \left\|T_{\bar{\lambda}}\left[T_{\lambda}\left[\Psi \mid X_{n}\right]\left(\cdot, \vec{\xi}_{n}\right) \mid X_{n}\right]\left(\cdot, \vec{\zeta}_{n}\right)-\Psi_{1}\left(\cdot, \vec{\zeta}_{n}+\vec{\xi}_{n}\right)\right\|_{p} \\
& \leq \int_{\mathbb{R}^{r}} \int_{L_{2}[0, t]}\left[1-H_{2}\left(\frac{|\lambda|^{2}}{2 \operatorname{Re} \lambda}, v, \vec{z}\right) H_{5}\left(\frac{|\lambda|^{2}}{2 \operatorname{Re} \lambda}, v, \vec{z}\right)\right] d|\sigma|(v) d|\rho|(\vec{z})
\end{aligned}
$$

which converges to 0 as $\lambda$ approaches to $-i q$ through $\mathbb{C}_{+}$by the dominated convergence theorem.

Theorem 4.3. Let $X_{n+1}$ be given by (1.3) and let $1 \leq p \leq \infty$. Furthermore, let $\Psi_{1}$ and $\Psi_{2}$ be as given in Theorem 2.3. Then for a nonzero real $q$, w $w_{\varphi}$-a.e. $y \in C[0, t]$ and $P_{X_{n+1}}$-a.e. $\vec{\xi}_{n+1}, \vec{\zeta}_{n+1} \in \mathbb{R}^{n+2}$,

$$
\begin{aligned}
& T_{q}^{(p)}\left[\left[\left(\Psi_{1} * \Psi_{2}\right)_{q} \mid X_{n+1}\right]\left(\cdot, \vec{\xi}_{n+1}\right) \mid X_{n+1}\right]\left(y, \vec{\zeta}_{n+1}\right) \\
& =\left[T_{q}^{(p)}\left[\Psi_{1} \mid X_{n+1}\right]\left(\frac{1}{\sqrt{2}} y, \frac{1}{\sqrt{2}}\left(\vec{\zeta}_{n+1}+\vec{\xi}_{n+1}\right)\right)\right] \\
& \quad \times\left[T_{q}^{(p)}\left[\Psi_{2} \mid X_{n+1}\right]\left(\frac{1}{\sqrt{2}} y, \frac{1}{\sqrt{2}}\left(\vec{\zeta}_{n+1}-\vec{\xi}_{n+1}\right)\right)\right] .
\end{aligned}
$$


Proof. Let $\lambda \in \mathbb{C}_{+}^{\sim}$. For $\lambda_{1}>0$, $w_{\varphi}$-a.e. $y \in C[0, t]$ and $P_{X_{n+1}}$-a.e. $\vec{\xi}_{n+1}, \vec{\zeta}_{n+1} \in \mathbb{R}^{n+2}$,

$$
\begin{aligned}
& I_{\left[\left(\Psi_{1} * \Psi_{2}\right)_{\lambda} \mid X_{n+1}\right]\left(\cdot, \vec{\xi}_{n+1}\right)}^{\lambda_{1}}\left(y, \vec{\zeta}_{n+1}\right) \\
& =\int_{\mathbb{R}^{r}} \int_{\mathbb{R}^{r}} \int_{L_{2}[0, t]} \int_{L_{2}[0, t]} \int_{C} H_{1}\left(\lambda_{1}^{-\frac{1}{2}}(x-[x])+y+\left[\vec{\zeta}_{n+1}\right], \vec{\xi}_{n+1},\right. \\
& \left.\frac{1}{\sqrt{2}} v_{1}, \frac{1}{\sqrt{2}} \vec{z}_{1}\right) H_{1}\left(\lambda_{1}^{-\frac{1}{2}}(x-[x])+y+\left[\vec{\zeta}_{n+1}\right],-\vec{\xi}_{n+1}, \frac{1}{\sqrt{2}} v_{2}, \frac{1}{\sqrt{2}} \vec{z}_{2}\right) \\
& \times H_{2}\left(\lambda, \frac{1}{\sqrt{2}}\left(v_{1}-v_{2}\right), \frac{1}{\sqrt{2}}\left(\vec{z}_{1}-\vec{z}_{2}\right)\right) d w_{\varphi}(x) d \sigma_{1}\left(v_{1}\right) d \sigma_{2}\left(v_{2}\right) d \rho_{1}\left(\vec{z}_{1}\right) d \rho_{2}\left(\vec{z}_{2}\right)
\end{aligned}
$$

by Theorem 2.3, where $H_{1}$ and $H_{2}$ are given by (2.3) and (2.4), respectively. Now, we have

$$
\begin{aligned}
I_{\left[\left(\Psi_{1} * \Psi_{2}\right)_{\lambda} \mid X_{n+1}\right]\left(\cdot, \vec{\xi}_{n+1}\right)}^{\lambda_{1}}\left(y, \vec{\zeta}_{n+1}\right) \\
=\int_{\mathbb{R}^{r}} \int_{\mathbb{R}^{r}} \int_{L_{2}[0, t]} \int_{L_{2}[0, t]} \int_{C} H_{1}\left(\frac{1}{\sqrt{2}} y, \frac{1}{\sqrt{2}}\left(\vec{\zeta}_{n+1}+\vec{\xi}_{n+1}\right), v_{1}, \vec{z}_{1}\right) \\
\times H_{1}\left(\frac{1}{\sqrt{2}} y, \frac{1}{\sqrt{2}}\left(\vec{\zeta}_{n+1}-\vec{\xi}_{n+1}\right), v_{2}, \vec{z}_{2}\right) H_{2}\left(\lambda, \frac{1}{\sqrt{2}}\left(v_{1}-v_{2}\right), \frac{1}{\sqrt{2}}\left(\vec{z}_{1}-\vec{z}_{2}\right)\right) \\
\times H_{3}\left(\lambda_{1}, \frac{1}{\sqrt{2}}\left(v_{1}+v_{2}\right), \frac{1}{\sqrt{2}}\left(\vec{z}_{1}+\vec{z}_{2}\right), x\right) \\
\quad d w_{\varphi}(x) d \sigma_{1}\left(v_{1}\right) d \sigma_{2}\left(v_{2}\right) d \rho_{1}\left(\vec{z}_{1}\right) d \rho_{2}\left(\vec{z}_{2}\right),
\end{aligned}
$$

where $H_{3}$ is given by (2.6). By Lemma 2.1, we obtain that

$$
\begin{aligned}
& I_{\left[\left(\Psi_{1} * \Psi_{2}\right)_{\lambda} \mid X_{n+1}\right]\left(\cdot, \vec{\xi}_{n+1}\right)}^{\lambda_{1}}\left(y, \vec{\zeta}_{n+1}\right) \\
& =\int_{\mathbb{R}^{r}} \int_{\mathbb{R}^{r}} \int_{L_{2}[0, t]} \int_{L_{2}[0, t]} H_{1}\left(\frac{1}{\sqrt{2}} y, \frac{1}{\sqrt{2}}\left(\vec{\zeta}_{n+1}+\vec{\xi}_{n+1}\right), v_{1}, \vec{z}_{1}\right) \\
& \times H_{1}\left(\frac{1}{\sqrt{2}} y, \frac{1}{\sqrt{2}}\left(\vec{\zeta}_{n+1}-\vec{\xi}_{n+1}\right), v_{2}, \vec{z}_{2}\right) H_{2}\left(\lambda, \frac{1}{\sqrt{2}}\left(v_{1}-v_{2}\right),\right. \\
& \left.\frac{1}{\sqrt{2}}\left(\vec{z}_{1}-\vec{z}_{2}\right)\right) H_{2}\left(\lambda_{1}, \frac{1}{\sqrt{2}}\left(v_{1}+v_{2}\right), \frac{1}{\sqrt{2}}\left(\vec{z}_{1}+\vec{z}_{2}\right)\right) d \sigma_{1}\left(v_{1}\right) \\
& d \sigma_{2}\left(v_{2}\right) d \rho_{1}\left(\vec{z}_{1}\right) d \rho_{2}\left(\vec{z}_{2}\right) .
\end{aligned}
$$

By (2.5), the Morera's theorem and the dominated convergence theorem, we have the analytic extension $\left.T_{\lambda_{1}}\left[\left(\Psi_{1} * \Psi_{2}\right)_{\lambda} \mid X_{n+1}\right]\left(\cdot, \vec{\xi}_{n+1}\right) \mid X_{n+1}\right](y$, $\left.\vec{\zeta}_{n+1}\right)$ of (4.2) as function of $\lambda_{1} \in \mathbb{C}_{+}$. Let $T_{q}^{(p)}\left[\left[\left(\Psi_{1} * \Psi_{2}\right)_{\lambda} \mid X_{n+1}\right](\cdot\right.$, $\left.\left.\vec{\xi}_{n+1}\right) \mid X_{n+1}\right]\left(y, \vec{\zeta}_{n+1}\right)$ be given by the right hand side of (4.2), where $\lambda_{1}$ 
is replaced by $-i q$, and let $\frac{1}{p}+\frac{1}{p^{\prime}}=1$. By $(2.5)$

$$
\begin{aligned}
& \| T_{\lambda_{1}}\left[\left[\left(\Psi_{1} * \Psi_{2}\right)_{\lambda} \mid X_{n+1}\right]\left(\cdot, \vec{\xi}_{n+1}\right) \mid X_{n+1}\right]\left(\cdot, \vec{\zeta}_{n+1}\right) \\
& \quad-T_{q}^{(p)}\left[\left[\left(\Psi_{1} * \Psi_{2}\right)_{\lambda} \mid X_{n+1}\right]\left(\cdot, \vec{\xi}_{n+1}\right) \mid X_{n+1}\right]\left(\cdot, \vec{\zeta}_{n+1}\right) \|_{p^{\prime}} \\
& \leq \int_{\mathbb{R}^{r}} \int_{\mathbb{R}^{r}} \int_{L_{2}[0, t]} \int_{L_{2}[0, t]} \mid H_{2}\left(\lambda_{1}, \frac{1}{\sqrt{2}}\left(v_{1}+v_{2}\right), \frac{1}{\sqrt{2}}\left(\vec{z}_{1}+\vec{z}_{2}\right)\right)- \\
& H_{2}\left(-i q, \frac{1}{\sqrt{2}}\left(v_{1}+v_{2}\right), \frac{1}{\sqrt{2}}\left(\vec{z}_{1}+\vec{z}_{2}\right)\right)|d| \sigma_{1}\left|\left(v_{1}\right) d\right| \sigma_{2}\left|\left(v_{2}\right) d\right| \rho_{1}\left|\left(\vec{z}_{1}\right) d\right| \rho_{2} \mid\left(\vec{z}_{2}\right)
\end{aligned}
$$

which converges to 0 as $\lambda_{1}$ approaches to $-i q$ through $\mathbb{C}_{+}$by the dominated convergence theorem. This shows the existence of $T_{q}^{(p)}\left[\left[\left(\Psi_{1} *\right.\right.\right.$ $\left.\left.\left.\Psi_{2}\right)_{\lambda} \mid X_{n+1}\right]\left(\cdot, \vec{\xi}_{n+1}\right) \mid X_{n+1}\right]\left(y, \vec{\zeta}_{n+1}\right)$. Now, we have by $(4.2)$

$$
\begin{aligned}
& T_{q}^{(p)}\left[\left[\left(\Psi_{1} * \Psi_{2}\right)_{q} \mid X_{n+1}\right]\left(\cdot, \vec{\xi}_{n+1}\right) \mid X_{n+1}\right]\left(y, \vec{\zeta}_{n+1}\right) \\
& =\int_{\mathbb{R}^{r}} \int_{\mathbb{R}^{r}} \int_{L_{2}[0, t]} \int_{L_{2}[0, t]} H_{1}\left(\frac{1}{\sqrt{2}} y, \frac{1}{\sqrt{2}}\left(\vec{\zeta}_{n+1}+\vec{\xi}_{n+1}\right), v_{1}, \vec{z}_{1}\right) H_{1}( \\
& \left.\left.\frac{1}{\sqrt{2}} y, \frac{1}{\sqrt{2}}\left(\vec{\zeta}_{n+1}-\vec{\xi}_{n+1}\right), v_{2}, \vec{z}_{2}\right) H_{2}\left(-i q, v_{1}, \vec{z}_{1}\right)\right) H_{2}\left(-i q, v_{2}, \vec{z}_{2}\right) \\
& d \sigma_{1}\left(v_{1}\right) d \sigma_{2}\left(v_{2}\right) d \rho_{1}\left(\vec{z}_{1}\right) d \rho_{2}\left(\vec{z}_{2}\right)
\end{aligned}
$$

which completes the proof by Theorem 2.2 .

Note that by the same method as used in the proof of Theorem 4.3, we can obtain (4.1), where $-i q$ is replaced by $\lambda \in \mathbb{C}_{+}$.

Now, we have the final result of our work.

THeOREM 4.4. If $X_{n+1}, \vec{\xi}_{n+1}$ and $\vec{\zeta}_{n+1}$ in Theorem 4.3 are replaced by $X_{n}, \vec{\xi}_{n} \in \mathbb{R}^{n+1}$ and $\vec{\zeta}_{n} \in \mathbb{R}^{n+1}$, respectively, then the conclusion given by (4.1) is still true.

Proof. Let $\lambda \in \mathbb{C}_{+}^{\sim}$. For $\lambda_{1}>0, w_{\varphi}$-a.e. $y \in C[0, t]$ and $P_{X_{n}}$-a.e. $\vec{\xi}_{n}, \vec{\zeta}_{n} \in \mathbb{R}^{n+1}$

$$
\begin{aligned}
& K_{\left[\left(\Psi_{1} * \Psi_{2}\right)_{\lambda} \mid X_{n}\right]\left(\cdot, \vec{\xi}_{n}\right)}^{\lambda_{1}}\left(y, \vec{\zeta}_{n}\right) \\
& =\int_{\mathbb{R}^{r}} \int_{\mathbb{R}^{r}} \int_{L_{2}[0, t]} \int_{L_{2}[0, t]} H_{1}\left(y, 0, \frac{1}{\sqrt{2}}\left(v_{1}+v_{2}\right), \frac{1}{\sqrt{2}}\left(\vec{z}_{1}+\vec{z}_{2}\right)\right) H_{2}\left(\lambda_{1},\right.
\end{aligned}
$$




$$
\begin{aligned}
& \left.\frac{1}{\sqrt{2}}\left(v_{1}+v_{2}\right), \frac{1}{\sqrt{2}}\left(\vec{z}_{1}+\vec{z}_{2}\right)\right) H_{4}\left(\vec{\zeta}_{n}, \frac{1}{\sqrt{2}}\left(v_{1}+v_{2}\right), \frac{1}{\sqrt{2}}\left(\vec{z}_{1}+\vec{z}_{2}\right)\right) H_{5} \\
& \left(\lambda_{1}, \frac{1}{\sqrt{2}}\left(v_{1}+v_{2}\right), \frac{1}{\sqrt{2}}\left(\vec{z}_{1}+\vec{z}_{2}\right)\right) H_{2}\left(\lambda, \frac{1}{\sqrt{2}}\left(v_{1}-v_{2}\right), \frac{1}{\sqrt{2}}\left(\vec{z}_{1}-\vec{z}_{2}\right)\right) \\
& H_{4}\left(\vec{\xi}_{n}, \frac{1}{\sqrt{2}}\left(v_{1}-v_{2}\right), \frac{1}{\sqrt{2}}\left(\vec{z}_{1}-\vec{z}_{2}\right)\right) H_{5}\left(\lambda, \frac{1}{\sqrt{2}}\left(v_{1}-v_{2}\right), \frac{1}{\sqrt{2}}\left(\vec{z}_{1}-\vec{z}_{2}\right)\right) \\
& d \sigma_{1}\left(v_{1}\right) d \sigma_{2}\left(v_{2}\right) d \rho_{1}\left(\vec{z}_{1}\right) d \rho_{2}\left(\vec{z}_{2}\right)
\end{aligned}
$$

by Lemma 3.1, Theorems 3.2 and 3.3, where $H_{1}, H_{2}, H_{4}$ and $H_{5}$ are given by (2.3), (2.4), (3.1) and (3.2), respectively. By (2.5), the Morera's theorem and the dominated convergence theorem, we have the analytic extension $T_{\lambda_{1}}\left[\left[\left(\Psi_{1} * \Psi_{2}\right)_{\lambda} \mid X_{n}\right]\left(\cdot, \vec{\xi}_{n}\right) \mid X_{n}\right]\left(y, \vec{\zeta}_{n}\right)$ of $K_{\left[\left(\Psi_{1} * \Psi_{2}\right)_{\lambda} \mid X_{n}\right]\left(\cdot, \vec{\xi}_{n}\right)}^{\lambda_{1}}(y$, $\left.\vec{\zeta}_{n}\right)$ as function of $\lambda_{1} \in \mathbb{C}_{+}$. Let $T_{q}^{(p)}\left[\left[\left(\Psi_{1} * \Psi_{2}\right)_{\lambda} \mid X_{n}\right]\left(\cdot, \vec{\xi}_{n}\right) \mid X_{n}\right]\left(y, \vec{\zeta}_{n}\right)$ be given by the right-hand side of the above equality, where $\lambda_{1}$ is replaced by $-i q$, and let $\frac{1}{p}+\frac{1}{p^{\prime}}=1$. Then we have

$$
\begin{aligned}
& \| T_{\lambda_{1}}\left[\left[\left(\Psi_{1} * \Psi_{2}\right)_{\lambda} \mid X_{n}\right]\left(\cdot, \vec{\xi}_{n}\right) \mid X_{n}\right]\left(\cdot, \vec{\zeta}_{n}\right) \\
& -T_{q}^{(p)}\left[\left[\left(\Psi_{1} * \Psi_{2}\right)_{\lambda} \mid X_{n}\right]\left(\cdot, \vec{\xi}_{n}\right) \mid X_{n}\right]\left(\cdot, \vec{\zeta}_{n}\right) \|_{p^{\prime}} \\
& \leq \int_{\mathbb{R}^{r}} \int_{\mathbb{R}^{r}} \int_{L_{2}[0, t]} \int_{L_{2}[0, t]} \mid H_{2}\left(\lambda_{1}, \frac{1}{\sqrt{2}}\left(v_{1}+v_{2}\right), \frac{1}{\sqrt{2}}\left(\vec{z}_{1}+\vec{z}_{2}\right)\right) \\
& \times H_{5}\left(\lambda_{1}, \frac{1}{\sqrt{2}}\left(v_{1}+v_{2}\right), \frac{1}{\sqrt{2}}\left(\vec{z}_{1}+\vec{z}_{2}\right)\right)-H_{2}\left(-i q, \frac{1}{\sqrt{2}}\left(v_{1}+v_{2}\right),\right. \\
& \left.\frac{1}{\sqrt{2}}\left(\vec{z}_{1}+\vec{z}_{2}\right)\right) H_{5}\left(-i q, \frac{1}{\sqrt{2}}\left(v_{1}+v_{2}\right), \frac{1}{\sqrt{2}}\left(\vec{z}_{1}+\vec{z}_{2}\right)\right) \mid \\
& d\left|\sigma_{1}\right|\left(v_{1}\right) d\left|\sigma_{1}\right|\left(v_{2}\right) d\left|\rho_{1}\right|\left(\vec{z}_{1}\right) d\left|\rho_{2}\right|\left(\vec{z}_{2}\right)
\end{aligned}
$$

which converges to 0 as $\lambda$ approaches to $-i q$ through $\mathbb{C}_{+}$by the dominated convergence theorem. This shows the existence of $T_{q}^{(p)}\left[\left[\left(\Psi_{1} *\right.\right.\right.$ $\left.\left.\left.\Psi_{2}\right)_{\lambda} \mid X_{n}\right]\left(\cdot, \vec{\xi}_{n}\right) \mid X_{n}\right]\left(\cdot, \vec{\zeta}_{n}\right)$. By $(2.3)$ and $(3.1)$, it is not difficult to show

$$
\begin{aligned}
& H_{1}\left(y, 0, \frac{1}{\sqrt{2}}\left(v_{1}+v_{2}\right), \frac{1}{\sqrt{2}}\left(\vec{z}_{1}+\vec{z}_{2}\right)\right) \\
& =H_{1}\left(\frac{1}{\sqrt{2}} y, 0, v_{1}, \vec{z}_{1}\right) H_{1}\left(\frac{1}{\sqrt{2}} y, 0, v_{2}, \vec{z}_{2}\right)
\end{aligned}
$$

and 


$$
\begin{aligned}
& H_{4}\left(\vec{\zeta}_{n}, \frac{1}{\sqrt{2}}\left(v_{1}+v_{2}\right), \frac{1}{\sqrt{2}}\left(\vec{z}_{1}+\vec{z}_{2}\right)\right) \\
& \times H_{4}\left(\vec{\xi}_{n}, \frac{1}{\sqrt{2}}\left(v_{1}-v_{2}\right), \frac{1}{\sqrt{2}}\left(\vec{z}_{1}-\vec{z}_{2}\right)\right) \\
& =H_{4}\left(\frac{1}{\sqrt{2}}\left(\vec{\zeta}_{n}+\vec{\xi}_{n}\right), v_{1}, \vec{z}_{1}\right) H_{4}\left(\frac{1}{\sqrt{2}}\left(\vec{\zeta}_{n}-\vec{\xi}_{n}\right), v_{2}, \vec{z}_{2}\right) .
\end{aligned}
$$

Furthermore, by (2.4) and (3.2),

$$
\begin{aligned}
& H_{2}\left(\lambda, \frac{1}{\sqrt{2}}\left(v_{1}+v_{2}\right), \frac{1}{\sqrt{2}}\left(\vec{z}_{1}+\vec{z}_{2}\right)\right) \\
& \times H_{2}\left(\lambda, \frac{1}{\sqrt{2}}\left(v_{1}-v_{2}\right), \frac{1}{\sqrt{2}}\left(\vec{z}_{1}-\vec{z}_{2}\right)\right)=H_{2}\left(\lambda, v_{1}, \vec{z}_{1}\right) H_{2}\left(\lambda, v_{2}, \vec{z}_{2}\right)
\end{aligned}
$$

and

$$
\begin{aligned}
& H_{5}\left(\lambda, \frac{1}{\sqrt{2}}\left(v_{1}+v_{2}\right), \frac{1}{\sqrt{2}}\left(\vec{z}_{1}+\vec{z}_{2}\right)\right) \\
& \times H_{5}\left(\lambda, \frac{1}{\sqrt{2}}\left(v_{1}-v_{2}\right), \frac{1}{\sqrt{2}}\left(\vec{z}_{1}-\vec{z}_{2}\right)\right)=H_{5}\left(\lambda, v_{1}, \vec{z}_{1}\right) H_{5}\left(\lambda, v_{2}, \vec{z}_{2}\right) .
\end{aligned}
$$

Now, we have the result by Theorem 3.2 .

Note that by the same method as used in the proof of Theorem 4.4, we can show the same equality in the above theorem, where $-i q$ is replaced by $\lambda \in \mathbb{C}_{+}$.

\section{References}

[1] R. H. Cameron and D. A. Storvick, Some Banach algebras of analytic Feynman integrable functionals, Lecture Notes in Mathematics 798, Springer, BerlinNew York, 1980.

[2] K. S. Chang, D. H. Cho, B. S. Kim, T. S. Song and I. Yoo, Conditional FourierFeynman transform and convolution product over Wiener paths in abstract Wiener space, Integral Transforms Spec. Funct. 14 (2003), no. 3, 217-235.

[3] S. J. Chang and D. Skoug, The effect of drift on conditional Fourier-Feynman transforms and conditional convolution products, Int. J. Appl. Math. 2 (2000), no. 4, 505-527.

[4] D. H. Cho, A time-independent conditional Fourier-Feynman transform and convolution product on an analogue of Wiener space, Honam J. Math. (2013), to appear.

[5] D. H. Cho, A time-dependent conditional Fourier-Feynman transform and convolution product on an analogue of Wiener space, Houston J. Math. (2012), submitted. 
[6] D. H. Cho, A conditional integral transform and conditional convolution product on a function space, Integral Transforms Spec. Funct. 23 (2012), no 6, 405-420.

[7] D. H. Cho, A simple formula for an analogue of conditional Wiener integrals and its applications II, Czechoslovak Math. J. 59 (2009), no. 2, 431-452.

[8] D. H. Cho, A simple formula for an analogue of conditional Wiener integrals and its applications, Trans. Amer. Math. Soc. 360 (2008), no. 7, 3795-3811.

[9] D. H. Cho, Conditional Fourier-Feynman transform and convolution product over Wiener paths in abstract Wiener space: an $L_{p}$ theory, J. Korean Math. Soc. 41 (2004), no. 2, 265-294.

[10] D. H. Cho, B. J. Kim and I. Yoo, Analogues of conditional Wiener integrals and their change of scale transformations on a function space, J. Math. Anal. Appl. 359 (2009), no. 2, 421-438.

[11] M. K. Im and K. S. Ryu, An analogue of Wiener measure and its applications, J. Korean Math. Soc. 39 (2002), no. 5, 801-819.

[12] M. J. Kim, Conditional Fourier-Feynman transform and convolution product on a function space, Int. J. Math. Anal. 3 (2009), no. 10, 457-471.

[13] K. S. Ryu and M. K. Im, A measure-valued analogue of Wiener measure and the measure-valued Feynman-Kac formula, Trans. Amer. Math. Soc. 354 (2002), no. $12,4921-4951$.

[14] K. S. Ryu, M. K. Im, and K. S. Choi, Survey of the theories for analogue of Wiener measure space, Interdiscip. Inform. Sci. 15 (2009), no. 3, 319-337.

Department of Mathematics

Kyonggi University

Suwon 443-760, Republic of Korea

E-mail: j94385@kyonggi.ac.kr 